\title{
Identification of the risk for liver fibrosis on CHB patients using an artificial neural network based on routine and serum markers
}

\author{
Danan Wang, Qinghui Wang, Fengping Shan, Beixing Liu, Changlong Lu*
}

\begin{abstract}
Background: Liver fibrosis progression is commonly found in patients with CHB. Liver biopsy is a gold standard for identifying the extent of liver fibrosis, but has many draw-backs. It is essential to construct a noninvasive model to predict the levels of risk for liver fibrosis. It would provide very useful information to help reduce the number of liver biopsies of CHB patients.

Methods: 339 chronic hepatitis B patients with HBsAg-positive were investigated retrospectively, and divided at random into 2 subsets with twice as many patients in the training set as in the validation set; 116 additional patients were consequently enrolled in the study as the testing set. A three-layer artificial neural network was developed using a Bayesian learning algorithm. Sensitivity and ROC analysis were performed to explain the importance of input variables and the performance of the neural network.
\end{abstract}

Results: There were 329 patients without significant fibrosis and 126 with significant fibrosis in the study. All markers except gender, HB, ALP and TP were found to be statistically significant factors associated with significant fibrosis. The sensitivity analysis showed that the most important factors in the predictive model were age, AST, platelet, and GGT, and the influence on the output variable among coal miners were 22.3-24.6\%. The AUROC in 3 sets was $0.883,0.884$, and 0.920 . In the testing set, for a decision threshold of 0.33 , sensitivity and negative predictive values were $100 \%$ and all CHB patients with significant fibrosis would be identified.

Conclusions: The artificial neural network model based on routine and serum markers would predict the risk for liver fibrosis with a high accuracy. $47.4 \%$ of CHB patients at a decision threshold of 0.33 would be free of liver biopsy and wouldn't be missed.

\section{Background}

Approximately 350 million people worldwide are chronically infected with hepatitis B virus (HBV), especially in many developing countries in Asia[1-3]. Liver fibrosis progression is commonly found in patients with chronic hepatitis B (CHB). Up to $40 \%$ of CHB patients will develop complications of liver cirrhosis and hepatocellular carcinoma (HCC)[4]. Patients with significant fibrosis or cirrhosis should be considered for antiviral therapy, which can potentially reverse cirrhosis and reduce complications[5-7].

\footnotetext{
* Correspondence: cllu@mail.cmu.edu.cn
Institute of Immunology, China Medical University, Shenyang, Liaoning,

* Correspondence: cllu@mail.cmu.edu.cn
Institute of Immunology, China Medical University, Shenyang, Liaoning, China
}

(c) 2010 Wang et al; licensee BioMed Central Ltd. This is an Open Access article distributed under the terms of the Creative Commons Attribution License (http://creativecommons.org/licenses/by/2.0), which permits unrestricted use, distribution, and reproduction in any medium, provided the original work is properly cited. the examination of the extent of liver fibrosis and HCC in patients with $\mathrm{CHB}$, has many draw-backs, such as pneumothorax, pain, hemorrhage, or puncture of other viscera[8-10]. In addition, sampling error leads to only $65 \%$ accuracy in liver biopsy specimens[11]. Therefore, there is an increasing demand for noninvasive predictive models of liver fibrosis. Those methods could predict the risk probability of liver fibrosis and identify $\mathrm{CHB}$ patients at high risk for liver fibrosis. If we only perform liver biopsy on $\mathrm{CHB}$ patients at high risk for liver fibrosis, the number of liver biopsies would be reduced. In the past few years, noninvasive biochemical markers of liver fibrosis which could respond of the chronically injured liver had made considerable progression[12-14]. Multiple algorithms based on a combination of fairly 
routine parameters had been repeatedly suggested [15-17]. However, most of them required non-routine laboratory assays or used complex and patented models so that their regular use was limited. In fact, the true usefulness of any indices or models for application to clinical settings should be the easiness of the procedure and analytical simplicity, so that the results can be compared between laboratories for a long period[18].

The complete blood count and liver function are routine tests on CHB patients with hepatitis B surface antigen (HBsAg-positive) in the clinical treatment. It is easy to measure those markers with low cost in some laboratories, even in the Community Hospitals. Moreover, the artificial neural network (ANN) is potentially more successful than a traditional statistical model in predicting clinical outcome $[19,20]$. It can build nonlinear statistical models through learning examples and has been widely applied to predict, diagnose, and classify disease in many fields $[21,22]$. In our study, we designed a neural network which used a Bayesian learning algorithm by introducing probabilistic treatment of the Bayesian inference technique. It can overcome some difficult problems, such as local trapping, over-fitting, and overtime in training. Also, it is proposed to have significant advantages over the conventional neural network approach [23].

The present study was performed on HBsAg-positive patients. An artificial neural network model based on routine and serum markers was constructed to predict the risk for liver fibrosis. The objective of this study was to determine the feasibility of these indices of routine and serum markers to predict the risk for liver fibrosis. Furthermore, we aimed to similarly validate the probability value in order to identify the risk for liver fibrosis on $\mathrm{CHB}$ patients and classify high risk groups.

\section{Methods}

\section{Patients}

In April 2008, all HBsAg-positive in-patients who had liver biopsy between January 2006 and March 2008 were retrospectively investigated at the Hospital of Infectious Diseases. Only patients without the following conditions were included for the study: presence of other causes of liver disease such as chronic hepatitis $\mathrm{C}(\mathrm{CHC})$, or hepatitis $\mathrm{E}$ (HE), etc., acute hepatitis, hepatocellular carcinoma, prior liver transplantation, insufficient liver tissue for staging of fibrosis, and incomplete data on complete blood count or some serum markers of fibrosis. Additionally, patients without routine and serum markers prior to drug treatment were also excluded. Within a week of liver biopsy, those markers were recorded. If more than one set of laboratory results were available, the set of results closest to the time of biopsy were used. Later, additional in-patients who met the aforementioned criteria were consequently enrolled in the study from April 2008 to July 2009.

The enrolled patients were informed all procedures which conformed to the Helsinki Declaration. The study procedures were also approved by the Ethics Committee of the Infectious Diseases Hospital of Shenyang. The verbal informed consent for all patients was provided.

\section{Laboratory tests}

Clinical chemistry tests were performed using 7150 Analyzer (Hitachi, Japan), and the complete blood count were measured on Hematology Analyzer (Beckman Coulter 5diff, U.S.A.).

\section{Liver biopsy histology}

Liver biopsy was performed by automatic fare cut biopsy needle. Length and width of each sample were at least $10 \mathrm{~mm}$ and $1 \mathrm{~mm}$, respectively. Liver sample of patients who were enrolled since April 2008 would contain 2 or more portal spaces. A single pathologist who had no clinical information of patients evaluated all biopsy results. The level of fibrosis was measured according to the METAVIR system, which had previously been applied in other reports on $\mathrm{CHB}[24,25]$. Fibrosis was staged from F0 to F4: F0, no fibrosis; F1, portal fibrosis without septa; F2, few septa; F3, numerous septa without cirrhosis; and F4, cirrhosis. Generally, significant fibrosis was defined by F2, F3, or F4 stages; whereas F0 or F1 was considered as insignificant fibrosis.

\section{Statistical analysis}

All statistical analysis was performed using MATLAB Neural Network Toolbox (2006) and SPSS Version 11.5 (SPSS, Inc, Chicago, IL). Data are reported as median (minimum-maximum) unless otherwise stated. The relationship between variables and the presence of significant fibrosis was assessed. The Mann-Whitney U Test, or Kruskal-Wallis Test was used for continuous variables when appropriate. The Chi-square test was used for categorical variables, and Fisher's exact test when appropriate. Those variables found to be strongly correlated to the presence of significant fibrosis were used to build the ANN.

\section{Development of the neural network}

Patients between January 2006 and March 2008 were randomly divided into 2 subsets: training set and validation set, with twice as many patients in training set as in validation set. Patients since April 2008 formed the testing set. The three-layer neural network model was built and trained using a Bayesian learning algorithm, which had 1 output neuron ( 0 , insignificant fibrosis; 1 , significant fibrosis). To determine the optimal number of neurons in the intermediate layer, we randomly split all 
data into 5 subsets of equal size. For any given number of neurons, we trained the network on all but one subset, and tested it on the remaining one. We started from 6 neurons, and gradually increased the number of neurons. When there were more than 13 neurons, the performance of the trained neural network on output sample in the testing set began to deteriorate. Hence, the intermediate layer had 13 neurons in the current neural network model. The number of training epochs was set to 300 , the learning rate was 0.05 , and the training goal was set at 0.001 .

As the neural network can be overtrained to recognize specific cases in the training set and result in good performance in the training set but not in the testing set, the validation set was used to decide when to stop training in order to minimize the potential bias.

\section{Sensitivity analysis}

Neural network models have been long criticized for being black box solutions primarily because of their inability to generate interpretable parameters for each input variable. To mitigate this problem, sensitivity analysis was adapted to explain their inference mechanism [26]. In our study, each input variable to the network varied between the mean \pm standard deviation, while all others were fixed at their respective means, and the corresponding change was recorded as a percentage deviation in the output. It could help illustrate the effect of changing a single input variable on the network output.

\section{ROC curves}

The receive operating characteristic (ROC) methodology is a computational methodology which has a very important connection to the neural network applied to classification applications[27]. An important feature of the ROC curves is that they readily incorporate prevalence and misclassification cost factors in decision-making. In our study, the predictive accuracies of the neural network were tested by measuring the area under the ROC (AUROC). The optimal cutoff value to predict the absence or presence of significant fibrosis was chosen. Diagnostic accuracy was evaluated by calculating sensitivity, specificity, positive and negative predictive values (PPV and NPV), and Youden's index.

\section{Results}

\section{Patients characteristics}

From January 2006 to March 2008, 396 patients who showed evidence of HBsAg-positive and liver biopsy were investigated. Among them, 57 were excluded from this study because of the following reasons: 24 had HCV or HIV co-infection, 4 had acute hepatitis, 7 had hepatocellular carcinoma, and 22 lacked complete data on laboratory tests. The demographic and clinical characteristics were similar between those 57 excluded patients and the rest of 339 patients fulfilling the entry criteria. From April 2008, 116 CHB patients were considered for enrollment. So, a total of 455 patients were included in this study in the end. Of overall subjects, 174 (38.2\%) had no fibrosis (F0), 155 (34.1\%) had portal fibrosis (F1), 70 (15.4\%) had septal fibrosis (F2), 47 (10.3\%) had numerous septa (F3), and 9 (2.0\%) had cirrhosis (F4). Their mean age was $33.9 \pm 11.7$ years; 289 (63.5\%) were male, and 166 (36.5\%) were female. Table 1 shows the comparison between patients with and without significant fibrosis. All markers, except for gender, hematoglobin $(\mathrm{Hb})$, alkaline phosphatase (ALP) and total protein (TP), were found to be statistically significant factors associated with significant fibrosis, and were used to initially construct the artificial neural network.

There were 226 subjects in the training set; 113 subjects in the validation set; 116 ubjects in the testing set. The variables used to construct the artificial neural network were compared in three sets (Table 2). These markers didn't appear to be statistically significant.

\section{Sensitivity analysis}

The sensitivity analysis of 10 variables was outlined in Figure 1. The value shown for each input variable is a measure of its relative importance, with 0 representing a variable that has no effect on the prediction and 1 representing a variable that completely dominates the prediction. The horizontal axis is the input variables; the vertical axis is the percent change on the output variable. The most important factors in the predictive model were age, aspartate aminotransferase (AST), platelet, and $\gamma$-glutamyltransferase (GGT), and the influence on the output variance among $\mathrm{CHB}$ patients were $24.6 \%, 23.8 \%, 23.7 \%$ and $22.3 \%$, respectively.

\section{Accuracy of the ROC curves}

The performance of the neural network in predicting significant fibrosis in three sets was high, and the AUROC was as follows: 0.883 (in the training set), 0.884 (in the validation set), and 0.920 (in the testing set; Figure 2).

Sensitivity, specificity, positive and negative predictive values at different cutoff points in the testing set were shown in Table 3. For a decision threshold of 0.33, sensitivity and negative predictive values were $100 \%$, which indicated that this model could predict insignificant fibrosis with the highest accuracy. This procedure would have correctly identified $47.4 \%$ of CHB patients without significant fibrosis, who needn't proceed with liver biopsy.

\section{Discussion}

Liver fibrosis is characterized by the accumulation of an extracellular matrix (ECM), which distorts the hepatic architecture. Liver fibrosis progression could commonly 
Table 1 Comparing characteristics of patients with and without significant fibrosis

\begin{tabular}{|c|c|c|c|c|}
\hline Variables & $\begin{array}{l}\text { Patients without significant fibrosis (F0-1), } n= \\
329\end{array}$ & $\begin{array}{l}\text { Patients with significant fibrosis (F2-4), } n= \\
126\end{array}$ & $\begin{array}{c}\chi^{2} \\
\text { value }\end{array}$ & $P$ \\
\hline Age & $29(16-60)$ & $40(17-75)$ & 52.21 & $\begin{array}{c}< \\
0.0001\end{array}$ \\
\hline Gender(M/F) & $215 / 114$ & $74 / 52$ & 1.723 & 0.189 \\
\hline $\operatorname{WBC}\left(10^{9} / L\right)$ & $5.3(2.3-12.2)$ & $4.5(1.3-18.1)$ & 14.51 & 0.0001 \\
\hline $\operatorname{RBC}\left(10^{12} / L\right)$ & $4.8(3.5-4.0)$ & $4.5(2.6-5.7)$ & 9.47 & 0.002 \\
\hline $\mathrm{Hb}(\mathrm{g} / \mathrm{L})$ & $145(87-173)$ & $141(85-167)$ & 1.79 & 0.174 \\
\hline Platelet $\left(10^{9} / \mathrm{L}\right)$ & $184(46-350)$ & $136(13-246)$ & 80.24 & 0.0001 \\
\hline $\mathrm{ALT}(\mathrm{U} / \mathrm{L})$ & $72.0(6-471)$ & $174.1(11-536)$ & 11.37 & $<0.00$ \\
\hline $\mathrm{AST}(\mathrm{U} / \mathrm{L})$ & $15.2(11-341)$ & $63.4(11-413)$ & 28.43 & $\begin{array}{c}< \\
0.0001\end{array}$ \\
\hline GGT(u/L) & $24.9(6-473)$ & $66.5(8-327)$ & 62.14 & $<.0001$ \\
\hline$A L P(u / L)$ & $106(7-219)$ & $117(19-307)$ & 2.78 & 0.099 \\
\hline $\operatorname{ChE}(\mathrm{u} / \mathrm{L})$ & $8054(70-20427)$ & $6617(50-12648)$ & 41.28 & $\begin{array}{c}< \\
0.0001\end{array}$ \\
\hline $\mathrm{TP}(\mathrm{g} / \mathrm{L})$ & $72.3(49-88.4)$ & $72.1(40-88.3)$ & 0.442 & 0.501 \\
\hline Alb $(g / L)$ & $45.4(11.6-47.0)$ & $43.2(10.9-52.6)$ & 18.14 & $\begin{array}{c}< \\
0.0001\end{array}$ \\
\hline $\mathrm{TBIL}(\mu \mathrm{mol} / \mathrm{L})$ & 13.4(3-138) & $15.3(5.5-216)$ & 11.03 & $<.0001$ \\
\hline $\begin{array}{l}\text { Length of liver biopsy } \\
(\mathrm{mm})\end{array}$ & $14(10-21)$ & $12(11-19)$ & 1.290 & 0.256 \\
\hline Number of liver biopsy & $2.0(1.0-4.0)$ & $2.5(1.0-5.0)$ & 1.621 & 0.197 \\
\hline
\end{tabular}

Table 2 Comparing input and output variables in three sets

\begin{tabular}{|c|c|c|c|c|}
\hline Variables & Patients in the training set, $n=226$ & Patients in the validation set, $n=113$ & Patients in the testing set, $n=116$ & $\chi^{2}(p)$ \\
\hline Age(years) & $30(16-62)$ & $35(16-75)$ & $32(16-74)$ & $\begin{array}{c}1.741 \\
(0.419)\end{array}$ \\
\hline $\operatorname{WBC}\left(10^{9} / \mathrm{L}\right)$ & $5.4(1.3-18.1)$ & $5.5(2.5-16.8)$ & $5.4(3.0-11)$ & $\begin{array}{c}0.231 \\
(0.891)\end{array}$ \\
\hline $\operatorname{RBC}\left(10^{12} / L\right)$ & $4.7(2.6-4.0)$ & $4.7(3.6-5.7)$ & $4.6(3.7-5.6)$ & $\begin{array}{c}0.310 \\
(0.856)\end{array}$ \\
\hline $\begin{array}{l}\text { Platelet( }\left(10^{9} /\right. \\
\mathrm{L})\end{array}$ & 165(53-246) & $170(13-350)$ & $185(46-345)$ & $\begin{array}{l}4.965 \\
(0.083)\end{array}$ \\
\hline $\operatorname{ALT}(U / L)$ & $87(16-471)$ & $80(11-536)$ & $72(6-507)$ & $\begin{array}{c}2.475 \\
(0.290)\end{array}$ \\
\hline $\mathrm{AST}(\mathrm{U} / \mathrm{L})$ & $48(11-413)$ & $42.5(15-375)$ & $39(11-361)$ & $\begin{array}{c}1.264 \\
(0.531)\end{array}$ \\
\hline GGT(U/L) & $29(6-421)$ & $33(6-288)$ & $34(10-473)$ & $\begin{array}{c}3.247 \\
(0.197)\end{array}$ \\
\hline $\operatorname{ChE}(\mathrm{U} / \mathrm{L})$ & $8402(50-20427)$ & 7674(145-11374) & $7258(360-12648)$ & $\begin{array}{c}3.051 \\
(0.217)\end{array}$ \\
\hline $\operatorname{Alb}(g / L)$ & $44.8(10.9-47.0)$ & $44.2(11.8-50.7)$ & $44.1(31.3-52.6)$ & $\begin{array}{c}0.412 \\
(0.814)\end{array}$ \\
\hline $\operatorname{TBIL}(\mu \mathrm{mol} / \mathrm{L})$ & 14.1(3.6-216) & 15.3(3-196) & 13.2(3- 134) & $\begin{array}{c}3.650 \\
(0.161)\end{array}$ \\
\hline $\begin{array}{c}\text { Fibrosis } \\
F(0-1) / F(2-4)\end{array}$ & $166 / 60$ & $83 / 30$ & $80 / 36$ & $\begin{array}{c}0.657 \\
(0.720)\end{array}$ \\
\hline
\end{tabular}




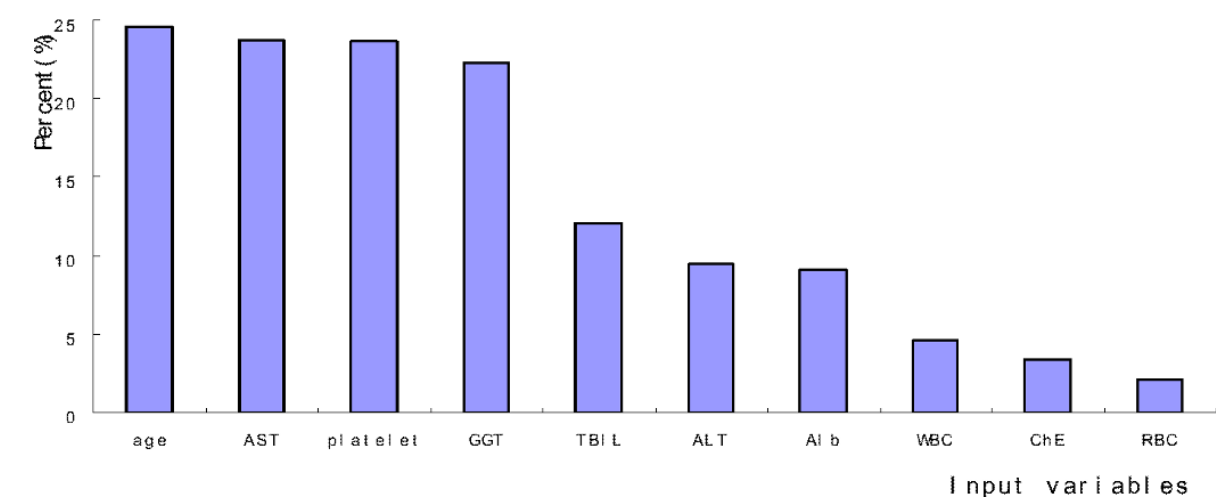

Figure 1 Sensitivity analysis of input variables. The horizontal axis is the input variables; the vertical axis is the percent change on the output variable. The value shown for each input variable is a measure of its relative importance, with 0 representing a variable that has no effect on the prediction and 1 representing a variable that completely dominates the prediction.

be found in chronic hepatitis B patients after an extensive period of time during which liver biochemical indices are found to be predominately or even persistently abnormal[28]. Due to the limitation of liver biopsy, noninvasive evaluation of liver fibrosis is thus of great clinical interests in order to assess the risk for liver fibrosis dynamically, or identify and monitor the patients who should be considered antiviral or other types of therapy.

Age, platelet, AST, ALT, GGT, etc., as routine biochemical markers, had been well known predictors of significant liver fibrosis[14,29,30]. In the present study, based on common biochemical parameters including routine and serum markers, we constructed a threelayer neural network which extended a back-propagation learning algorithm by introducing probabilistic treatment of the Bayesian inference technique for the synaptic weight[31]. Results of sensitivity analysis showed the importance of various predictors. As could be seen, the most important variables influencing the prediction of significant liver fibrosis were age, AST, platelet and GGT. These results were consistent with some of the earlier studies[14,16]. Sensitivity analysis showed that the neural network using Bayesian approaches could achieve its predictive purpose.

As demonstrated by ROC curves, the predictive accuracy of the artificial neural network was reasonably high in the training, validation, and testing sets. The AUROC were $0.883,0.884$ and 0.920 , respectively. In view with some noninvasive parameters directly or indirectly related to fibrogenesis, the most prevalent are the Fibrotest and the Actitest for necro-inflammator activity [32]. They are based on GGT, TBIL, haptoglobin, $\alpha 2-$ macroglobulin, apolipoprotein A1, and for the Actitest additionally on ALT[33]. Previous studies on Fibrotest and Actitest have been validated with the ranges of the AUROC of 0.75- 0.89 in CHB patients[14,25,32,34]. Although the two indices could provide better predictive values according to different criteria, they were calculated with a patented and complicated algorithm, and it

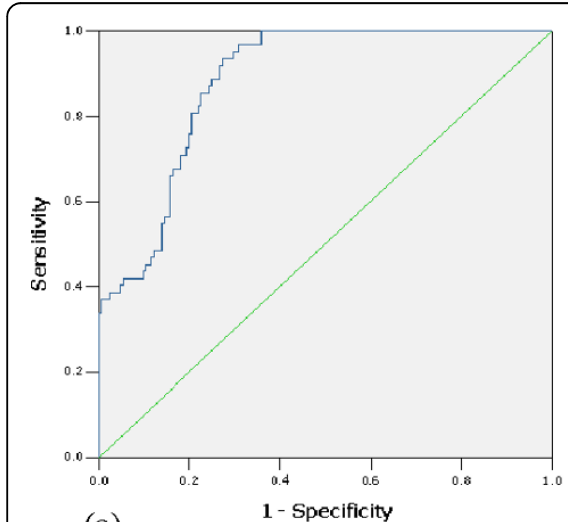

(a)

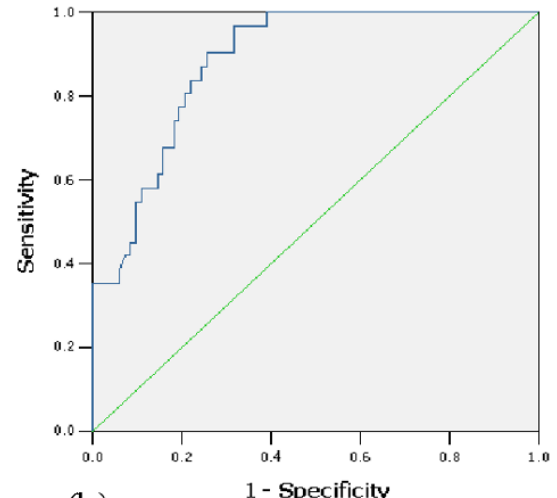

(b)

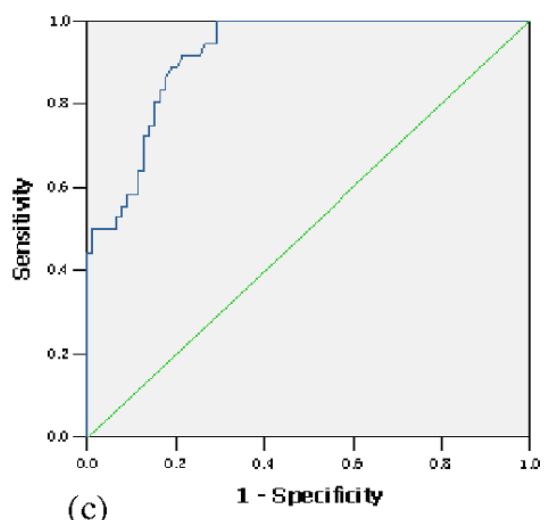

(c)

Figure 2 ROC curve of the neural network output in three sets. (a) In the training set(Cutoff $=0.415$, AUROC $=0.883$ ); (b) In the validation $\operatorname{set}(C u t o f f=0.421$, AUROC $=0.884)$; (c) In the testing set(Cutoff $=0.418$, AUROC $=0.920$ ) 
Table 3 ROC analysis of artificial neural network output at different cutoff points in the testing set

\begin{tabular}{cccccc}
\hline $\begin{array}{c}\text { Cutoff } \\
\text { point }\end{array}$ & $\begin{array}{c}\text { Sensitivity } \\
\%\end{array}$ & $\begin{array}{c}\text { Specificity } \\
\%\end{array}$ & $\begin{array}{c}\text { PPV } \\
\%\end{array}$ & $\begin{array}{c}\text { NPV } \\
\%\end{array}$ & $\begin{array}{c}\text { Youden Index } \\
\%\end{array}$ \\
\hline 0.33 & 100.0 & 68.7 & 59.0 & 100.0 & 68.7 \\
0.35 & 94.4 & 75.0 & 63.0 & 96.8 & 69.4 \\
0.40 & 91.7 & 80.0 & 65.3 & 94.0 & 71.7 \\
0.45 & 83.3 & 85.0 & 71.4 & 91.9 & 68.3 \\
0.50 & 66.7 & 88.7 & 72.7 & 85.5 & 47.1 \\
0.55 & 55.5 & 92.5 & 76.9 & 82.2 & 44.2 \\
0.60 & 44.4 & 96.2 & 83.3 & 78.6 & 42.3 \\
0.70 & 36.1 & 100.0 & 100.0 & 77.7 & 36.1 \\
\hline
\end{tabular}

was difficult for physicians to use them to identify the states of liver fibrosis.

Hui AY constructed and validated a multivariate logistic regression model using body mass index, platelet, Alb, and TBIL level to predict advanced fibrosis, and the AUROC were 0.765-0.803[17]. Zeng MD also constructed a scoring system with forward logistic regression, which was expressed by the following formula[16]: $-13.995+3.22 \lg \left(\alpha_{2}\right.$-macroglobulin $)+3.096 \lg ($ age $)+$ $2.254 \lg (\mathrm{GGT})+2.437 \lg (\mathrm{HA})$. The AUROC of this model in the training and validation groups were 0.84 and 0.77 , respectively. The logistic regression, a generalized linear model used for binomial regression, is used for prediction of the probability of occurrence of an event by fitting data to a logistic curve. The artificial neural networks, a non-linear statistical data modeling tool, can be used to model complex relationships between inputs and outputs. Therefore, the present study provided evidence that the three-layer neural network model based on routine and serum markers was superior to other indices or models for identification of individual with or without significant fibrosis.

In our study, there were 126 patients with significant liver fibrosis, which only accounted for $27.7 \%$ of 455 CHB patients with liver biopsy. In other words, 329 (72.3\%) patients without significant liver fibrosis had undergone liver biopsy, and they could bear the damages from such an invasive procedure. From a more practical point of view, we wanted to reduce the number of liver biopsy procedures and also identify all $\mathrm{CHB}$ patients with significant fibrosis. Therefore, our study evaluated the influence of the different cutoff points on the accuracy of ROC. When we chose a high cutoff point, the number of $\mathrm{CHB}$ patients at high risk for significant liver fibrosis was few, and also fewer patients needed further liver biopsy or other examinations. But, there was a low sensitivity and many $\mathrm{CHB}$ patients with significant liver fibrosis could be missed. The purpose of predicting the state of liver fibrosis is to identify $\mathrm{CHB}$ patients with significant liver fibrosis or at high risk for liver fibrogenesis to prevent them from further liver fibrogenesis. Thus, we should choose a lower cutoff point to improve the sensitivity, and to reduce the number of missed $\mathrm{CHB}$ patients at high risk for liver fibrogenesis. So, we considered a probability value of 0.33 as a cutoff value. $\mathrm{CHB}$ patients with a probability value > 0.33 were considered with significant liver fibrosis or at high risk for liver fibrogenesis. In our study, all $\mathrm{CHB}$ patients with significant liver fibrosis would be identified. $47.4 \%$ (55/116) of the CHB patients would be free of liver biopsy and also wouldn't be missed.

\section{Limitations of the study}

Our study was conducted in a specialized hospital for infectious diseases. All individuals investigated were inpatients, therefore not a completely random sample of all $\mathrm{CHB}$ patients. However, owing to the limitation of liver biopsy, we thought that the predictive model in this study might be applied to identify the risk of liver fibrosis as some studies showed [35].

The accuracy of a test could vary with the definition of the target condition[36]. In our study, the occurrence of F2, F3, or F4 was considered as significant fibrosis so that the prevalence of significant fibrosis was relatively low, which could affect the diagnostic accuracy of the model by AUROC. The DANA (difference of prevalence of advanced and nonadvanced fibrosis stages) was used for the standardization of AUROC of fibrosis marker or model[37]. But comparing with other studies $[14,16,17,25,32]$, we didn't correct the AUROC by the DANA.

\section{Conclusions}

The study highlighted the construction and assessment of an artificial neural network for identifying the risk for liver fibrosis. Age, platelet, AST, ALT, GGT, etc., as the input variables of the artificial neural network model, were widely available. The results of our study showed that the three-layer artificial neural networks could effectively identify the risk for liver fibrosis in $\mathrm{CHB}$ patients with positive HBsAg. It could improve patient compliance, and reduce the need for liver biopsy required prior to antiviral or other types of therapy.

\footnotetext{
Abbreviations

HBV: hepatitis B virus; CHB: chronic hepatitis B; HCC: hepatocellular; ANN: artificial neural network; HBsAg-positive: hepatitis B surface antigen; CHC: chronic hepatitis $C_{;}$HE -hepatitis E; HCV: hepatitis C virus; HIV: human immunodeficiency virus; ROC: receive operating characteristic; AUROC: area under ROC; PPV: positive predictive values; NPV: negative predictive values; ECM: carcinoma extracellular matrix; WBC: white blood cell; RBC: red blood cell; Hb: hematoglobin; ALT: alanine aminotransferase; AST: aspartate aminotransferase; GGT: $\gamma$-glutamyltransferase; ALP: alkaline phosphatase; ChE: Cholinesterase; TP: total protein; Alb: albumin; TBIL: total bilirubin
} 


\section{Acknowledgements}

This work was supported by a grant from Liaoning Provincial Natural Science Foundation (No.20072101).

\section{Authors' contributions}

DW conducted the study, participated in the data collection and wrote the initial draft and revised the manuscripts. QW collected the preliminary data, and helped to draft the manuscript. FS participated in the study design and interpretation of the data. BL participated in data collection and gave inputs to the drafting of the manuscript. $C L$ helped to coordinate the study and the drafting and revisions of the paper. All co-authors read and approved the final manuscript.

\section{Competing interests}

The authors declare that they have no competing interests.

Received: 15 December 2009 Accepted: 24 August 2010

Published: 24 August 2010

\section{References}

1. Lavanchy D: Hepatitis B virus epidemiology, disease burden, treatment, and current and emerging prevention and control measures. J Viral Hepat 2004, 11:97-107.

2. Alam MM, Zaidi SZ, Malik SA, Naeem A, Shaukat S, Sharif S, Angez M, Khan A, Butt JA: Serology based disease status of Pakistani population infected with Hepatitis B virus. BMC Infect Dis 2007, 7:64.

3. Zeng Z, Guan L, An P, Sun S, O'Brien SJ, Winkler CA, HBV study consortium: A population-based study to investigate host genetic factors associated with hepatitis $B$ infection and pathogenesis in the Chinese population. BMC Infect Dis 2008, 8:1.

4. Maddrey W: Hepatitis B: an important public health issue. J Med Virol 2000, 61:362-366.

5. Wei L: Natural history of chronic hepatitis B virus infection: what determines prognosis after cirrhotic decompensation? J Gastroenterol Hepatol 2008, 23:1631-1632.

6. Liaw YF, Leung N, Guan R, Lau GK, Merican I, McCaughan G, Gane E, Kao JH, Omata M, Asian-Pacific consensus update working party on chronic hepatitis B: Asian-Pacific consensus ststement on the management of chronic hepatitis B: a 2005 update. Liver Int 2005, 25:472-489.

7. Liaw YF, Sung JJ, Chow WC, Farrell G, Lee CZ, Yuen H, Tanwandee T, Tao QM, Shue K, Keene ON, Dixon JS, Gray DF, Sabbat J, Cirrhosis Asian Lamivudine Multicentre Study Group: Lamivudine for patients with chronic hepatitis B and advanced liver disease. N Engl J Med 2004, 351:1521-1531.

8. Bravo AA, Sheth SG, Chopra S: Liver biopsy. N Engl J Med 2001 344:495-500

9. Narasimhan G, Sargios TN, Kalakuntla R, Homel P, Clain DJ, Theise ND, Bodenheimer HC Jr, Min AD: Treatment rates in patients with chronic hepatitis C after liver biopsy. J Viral Hepat 2006, 13:783-786.

10. Piccinino F, Sagnelli E, Pasquale G, Giusti G: Complications following percutaneous liver biopsy: A multicentre retrospective study on 68,276 biopsies. J Hepatol 1986, 2:165-173.

11. Siddique I, El-Naga HA, Madda JP, Memon A, Hasan F: Sampling variability on percutaneous liver biopsy in patients with chronic hepatitis $C$ virus infection. Scand J Gastroenterol 2003, 38:427-432.

12. Wai CT, Cheng CL, Wee A, Dan YY, Chan E, Chua W, Mak B, Oo AM, Lim SG: Non-invasive models for predicting histology in patients with chronic hepatitis B. Liver Int 2006, 26:666-672.

13. Mohamadnejad M, Montazeri G, Fazlollahi A, Zamani F, Nasiri J, Nobakht H, Forouzanfar MH, Abedian S, Tavangar SM, Mohamadkhani A, Ghoujeghi F, Estakhri A, Nouri N, Farzadi Z, Najjari A, Malekzadeh R: Noninvasive markers of liver fibrosis and inflammation in chronic hepatitis B-virus related liver disease. Am J Gastroenterol 2006, 101:2537-2545.

14. Fung J, Lai CL, Fong DY, Yuen JC, Wong DK, Yuen MF: Correlation of liver biochemistry with liver stiffess in chronic hepatitis $B$ and development of a predictive model for liver fibrosis. Liver International 2008 , 28:1408-1416.

15. Ngo $Y$, Benhamou $Y$, Thibault $V$, Ingiliz $P$, Munteanu $M$, Lebray $P$, Thabut $D$, Morra R, Messous D, Charlotte F, Imbert-Bismut F, Bonnefont-Rousselot D, Moussalli J, Ratziu V, Poynard T: An accurate definition of the status of inactive hepatitis $B$ virus carrier by a combination of biomarkers (FibroTest-ActiTest) and viral load. PloS One 2008, 2(3):e2573.

16. Zeng MD, Lu LG, Mao YM, Qiu DK, Li JQ, Wan MB, Chen CW, Wang JY, Cai $X$, Gao CF, Zhou XQ: Prediction of significant fibrosis in $\mathrm{HBeAg}$ positive patients with chronic hepatitis $B$ by a noninvasive model. Hepatology 2005, 42:1437-1445.

17. Hui AY, Chan HL, Wong WW, Liew CT, Chim AM, Chan FK, Sung JJ: Identification of chronic hepatitis $B$ patients without signification liver fibrosis by a simple noninvasive model. Am J Gastroenterol 2005, 100:616-623.

18. Lok AS, McMahon BJ: Chronic hepatitis B. Hepatology 2007, 45:507-539.

19. Green M, Bjork J, Forberg J, Ekelund U, Edenbrandt L, Ohlsson M: Comparison between neural networks and multiple logistic regression to predict acute coronary syndrome in the emergency room. Artif Intell Med 2006, 38:305-318.

20. Abbod MF, Catto JW, Linkens DA, Hamdy FC: Application of artificial intelligence to the management of urological cancer. J Urol 2007 178:1150-1156.

21. Wang CH, Mo LR, Lin RC, Kuo JJ, Chang KK, Wu JJ: Artificial neural network model is superior to logistic regression model in predicting treatment outcomes of interferon-based combination therapy in patients with chronic hepatitis C. Intervirology 2008, 51:14-20.

22. Piscaglia F, Cucchetti A, Benlloch S, Vivarelli M, Berenguer J, Bolondi L, Pinna $A D$, Berenguer M: Prediction of significant fibrosis in hepatitis $C$ virus infected liver transplant recipients by artificial neural network analysis of clinical factors. Eur J Gastroenterol Hepatol 2006, 18:1255-1261.

23. Lampinen J, Vehtati A: Bayesian approach for neural networks-review and case studies. Neural Netw 2001, 14:257-274.

24. Bedossa P, Poynard T: An algorithm for grading of activity in chronic hepatitis C. The METAVIR co-operative study group. Hepatology 1996, 24:289-293.

25. Myers RP, Tainturier MH, Ratziu V, Piton A, Thibault V, Imbert-Bismut F, Messous D, Charlotte F, Di Martino V, Benhamou Y, Poynard T: Prediction of liver histological lesions with biochemical markers in patients with chronic hepatitis B. J Hepatol 2003, 39:222-230.

26. Fish $\mathrm{KE}$, Blodgett JG: A visual method for determining variable importance in an artificial neural network model: an empirical benchmark study. J Targ Meas Anal Market 2003, 11:244-254.

27. Dayhoff JE, Deleo JM: Artificial neural network-Opening the black box. Cancer 2001, 91:1616-1635.

28. Dienstag JL, Goldin RD, Heathcote EJ, Hann HW, Woessner M, Stephenson SL, Gardner S, Gray DF, Schiff ER: Histological outcome during long-term lamivudine therapy. Gastroenterology 2003, 124:105-117.

29. Lin CS, Chang CS, Yang SS, Yeh HZ, Lin CW: Retrospective evaluation of serum markers APRI and AST/ALT for assessing liver fibrosis and cirrhosis in chronic hepatitis $B$ and $C$ patients with hepatocellular carcinoma. Internal Med 2008, 47:569-575.

30. Zhang $Y X$, Wu WJ, Zhang $Y Z$, Feng $Y L$, Zhou XX, Pan Q: Noninvasive assessment of liver fibrosis with combined serum aminotransferase/ platelet ratio index and hyaluronic acid in patients with chronic hepatitis B. World of Gastroenterol 2008, 14:7117-7121.

31. Yao XQ, Zhu HQ, She ZS: A dynamic Bayesian network approach to protein secondary structure prediction. BMC Bioinformatics 2008, 9:49-61.

32. Halfon $P$, Munteanu $M$, Poynard T: FibroTest-ActiTest as a non-invasive marker of liver fibrosis. Gastroenterol Clin Biol 2008, 32:22-39.

33. Poynard T, Imbert-Bismut F, Munteanu M, Messous D, Myers RP, Thabut D, Ratziu V, Mercadier A, Benhamou Y, Hainque B: Overview of the diagnostic value of biochemical markers of liver fibrosis (FibroTest, HCV FibroSure) and necrosis (ActiTest) in patients with chronic hepatitis C. Comp Hepatol 2004, 3:8-19.

34. Sebastiani G, Vario A, Guido M, Alberti A: Sequential algorithms combining non-invasive markers and biopsy for the assessment of liver fibrosis in chronic hepatitis B. World J Gastroenterol 2007, 13:525-731.

35. Shin WG, Park SH, Jang MK, Hahn TH, Kim JB, Lee MS, Kim DJ, Jun SY, Park CK: Aspartate aminotransferase to platelet ratio index (APRI) can predict liver fibrosis in chronic hepatitis B. Dig Liver Dis 2008, 40:267-274

36. Whiting P, Rutjes AW, Reitsma JB, Glas AS, Bossuyt PM, Kleijnen J: Sources of variation and bias in studies of diagnostic accuracy: a systematic review. Ann Intern Med 2004, 140:189-202

37. Poynard $T$, Halfon $P$, Castera L, Munteanu M, Imbert-Bismut F, Ratziu V, Benhamou Y, Bourliere M, de Ledinghen V, FibroPaca Group: 
Standardization of ROC Curve Areas for Diagnostic Evaluation of Liver Fibrosis Markers Based on Prevalences of Fibrosis Stages. Clinical

Chemistry 2007, 53:1615-1622.

\section{Pre-publication history}

The pre-publication history for this paper can be accessed here:

http://www.biomedcentral.com/1471-2334/10/251/prepub

doi:10.1186/1471-2334-10-251

Cite this article as: Wang et al:. Identification of the risk for liver fibrosis on CHB patients using an artificial neural network based on routine and serum markers. BMC Infectious Diseases 2010 10:251.

Submit your next manuscript to BioMed Central and take full advantage of:

- Convenient online submission

- Thorough peer review

- No space constraints or color figure charges

- Immediate publication on acceptance

- Inclusion in PubMed, CAS, Scopus and Google Scholar

- Research which is freely available for redistribution

Submit your manuscript at www.biomedcentral.com/submit
C Biomed Central 